Artículo original

\title{
Características del efecto Lau empleando redes de fase y de volumen fotorrefractivas
}

\author{
Gustavo Forte ${ }^{1,2, *}$, Myrian Tebaldi ${ }^{1,3}$, Nestor Bolognini ${ }^{1,2,3}$ \\ ${ }^{1}$ Centro de Investigaciones Ópticas, CIOp (CONICET La Plata-CIC-UNLP) La Plata, Argentina \\ ${ }^{2}$ Departamento de Física, Facultad de Ciencias Exactas, Universidad Nacional de La Plata. \\ ${ }^{3}$ UIDET OPTIMO, Departamento de Ciencias Básicas, Facultad de Ingeniería, Universidad Nacional de La Plata, Argentina
}

\begin{abstract}
Resumen
En este trabajo se estudia el efecto Lau empleando redes de fase de volumen generadas en un cristal fotorrefractivo tipo BSO. En nuestra propuesta la red de fase objeto se genera mediante la proyección de la imagen de una red iluminada incoherentemente en el volumen del cristal. Para esta red de fase fotorrefractiva se define un parámetro de modulación que depende del espesor del cristal. Se presentan resultados teóricos para diferentes distancias entre redes objeto-fuente que confirman nuestra propuesta. (C) 2018. Acad. Colomb. Cienc. Ex. Fis. Nat.
\end{abstract}

Palabras clave: Efecto Lau; Red de volumen; Cristales fotorrefractivos.

Features of the Lau effect by using a photorefractive volume phase grating

\begin{abstract}
This work analyzes the Lau effect produced by the volume phase grating generated in a photorefractive BSO crystal. In our proposal, the object phase grating is generated by projecting the image of an incoherently illuminated grating in the crystal volume. A modulation parameter that includes the effect of the crystal depth is introduced for this photorefractive phase grating. Theoretical results for different distances between object-source gratings that confirm our approach are presented. (C) 2018. Acad. Colomb. Cienc. Ex. Fis. Nat.
\end{abstract}

Key words: Lau effect; Volume grating; Photorefractive crystals.

\section{Introducción}

Es conocido que las autoimágenes de una estructura periódica desaparecen cuando se aumentan las dimensiones laterales de la fuente de iluminación (Patorski, 1989). Sin embargo, se pueden obtener patrones de franjas semejantes a las autoimágenes iluminando una red objeto con una fuente extensa convenientemente codificada. De este modo, empleando dos redes de amplitud separadas una distancia $z_{0}=\frac{n \cdot d^{2}}{\lambda}(n=1,2 \ldots)$ donde $d$ es el periodo de la red y $\lambda$ la longitud de onda de la fuente luminosa, se obtiene en el infinito franjas de alto contraste conocidas como franjas de Lau (Lau, 1948). La primera red de amplitud o red fuente de este dispositivo actúa codificando linealmente la fuente incoherente, mientras que la segunda red o red objeto, de amplitud o de fase, actúa como una transparencia objeto. Se han desarrollado diferentes modelos para explicar la formación de las franjas de Lau. Estos modelos se han basado en: la teoría de la difracción (Jahns y Lohmann, 1979), la teoría de la coherencia (Gori, 1979), la función de transferencia óptica del sistema (OTF) (Brenner, 1983), las funciones de Walsh (Colautti, 1988), las propiedades de un interferómetro de doble red (Swanson, 1982, Swanson, 1985), y la utilización de una representación dual espaciofase y de autoimágenes (Patorski, 1983).
El dispositivo de Lau convencional, que emplea dos redes de amplitud, posee una baja eficiencia luminosa debido a la absorción de luz que se produce en las redes de amplitud. Consecuentemente, las franjas de Lau presentan una baja visibilidad. El efecto Lau fue utilizado para implementar interferómetros ópticos con el propósito de obtener información de fase de un objeto bajo estudio (Tebaldi, 1999, Angel, 1999). Cabe destacar que, si bien este interferómetro presenta una mejor relación señal ruido que aquellos basados en el efecto Talbot, la baja visibilidad de las franjas limita el desarrollo de dispositivos basados en el efecto Lau. Por lo tanto, resulta relevante encontrar estrategias para obtener franjas de alto contraste. El empleo de una red objeto de fase produce una mejora en la luminosidad del dispositivo y la visibilidad de las franjas que depende de la modulación de fase de la red (Jinhong, 1987, Jinhong, 1988).

Los trabajos previos consideraban redes planas, en cambio en nuestra propuesta se estudia el efecto Lau empleando redes de fase de volumen generadas en un cristal

\footnotetext{
*Correspondencia:

Gustavo Forte, gforte@ciop.unlp.edu.ar

Recibido: 9 de febrero de 2018

Aceptado: 25 de abril de 2018

Editor: Román Castañeda Sepúlveda
} 
fotorrefractivo tipo BSO. Es evidente que, para poder aprovechar las características de volumen del medio de registro, es necesario que la distribución a ser almacenada sea tridimensional. En nuestra propuesta, la red objeto de fase se genera mediante la proyección de la imagen de una red de Ronchi iluminada incoherentemente en el volumen del cristal. Se emplea un cálculo basado en la OTF de un sistema formador de imagen incoherente para describir teóricamente la distribución de intensidad 3D. En Ref. (Forte, 2012) se determinaron las condiciones para generar una red en el volumen completo del cristal. Esta distribución se mapea en una perturbación del índice de refracción a través del efecto fotorrefractivo. Tres parámetros gobiernan la modulación de la red de índice: el campo eléctrico externo que determina la amplitud de la modulación, el diámetro de la pupila de salida del sistema de registro que controla la profundidad de la red a ser almacenada en el cristal y el espesor del cristal (Forte, 2012).

Para analizar teóricamente las franjas se utiliza un modelo basado en una formulación de la integral de camino para calcular la intensidad de los patrones generados por la red de fase de volumen (Forte, 2011). En nuestro cálculo se considera que la distribución de intensidad en el experimento de Lau puede ser interpretada como la correlación entre la distribución de intensidad de la red fuente, con el módulo cuadrado del patrón de Fresnel de la red objeto (Jahns y Lohmann, 1979, Forte, 2017). En nuestra propuesta se emplea un parámetro de fase, que incluye los efectos del volumen, respecto del cual la visibilidad mantiene la típica dependencia sinusoidal observada con redes planas. Este parámetro, propio de las redes de volumen tales como las generadas en cristales fotorrefractivos, incluye la dependencia con el espesor del cristal. En nuestro análisis elegimos adecuadamente el diámetro de la pupila del sistema óptico de registro con el propósito de garantizar la generación de una red en el volumen completo de los cristales. Se estudian patrones asociados a diferentes distancias entre redes fuenteobjeto. En cada caso, se determinan las condiciones para optimizar la visibilidad de las franjas a través de la elección adecuada del espesor del cristal.

\section{Planteamiento del problema y motivacion}

Como ya se mencionó en la introducción, se pueden obtener patrones de franjas de Lau iluminando una red objeto con una fuente extensa convenientemente codificada. En el dispositivo de Lau se obtienen franjas rectilíneas en el infinito o en el plano focal de una lente convergente (ver esquema experimental de la figura 1). Jahns y Lohmann (1979) aplicaron la teoría escalar de la difracción para demostrar que la distribución de intensidad en el experimento puede ser interpretada como la correlación entre la distribución de intensidad de la primera red o red fuente, $t(x)$, con el módulo cuadrado del patrón de Fresnel de la segunda red o red objeto, $\psi(x)$ :

$$
I(x)=|t(x)|^{2} \otimes|\psi(x)|^{2}
$$

donde $\otimes$ denota la operación de correlación. Las franjas obtenidas en este experimento empleando dos redes planas de amplitud presentan un contraste óptimo y un perfil triangular cuando las distancias entre las redes es $z_{0}=\frac{n \cdot d^{2}}{\lambda}$ $(n=1,2 \ldots)$ donde $d$ es el período de la red y $\lambda$ es la longitud de onda de la fuente de iluminación. El período $w$ del patrón de franjas de Lau obtenidas depende de $z_{0} \mathrm{y}$ está dado por $w=\frac{d \cdot f}{z_{0}}$, donde $f$ es la distancia focal de la lente.

El dispositivo de Lau que emplea redes de amplitud posee una baja eficiencia luminosa, debido a la absorción de luz que se produce en estas redes. El empleo de una red objeto de fase produce una mejora en la eficiencia luminosa. Considerando redes de perfil cuadradas para este caso, Tu Jinhong (1987) obtuvo que la distribución en el plano de observación está dada por:

$$
I(x)=2\left\{\frac{1}{4}+\sum_{n= \pm 1, \pm 3 \ldots}\left|C_{n}\right|^{2} \operatorname{sen}(m) \cdot \operatorname{sen}\left(\pi \lambda z_{0} n^{2} / d^{2}\right) \cdot \exp (i 2 n \pi x / w)\right\}
$$

donde $C_{\mathrm{n}}$ es el coeficiente de Fourier, $m$ es la modulación de la red de fase, $z_{0}$ es la distancia entre las redes fuente-objeto y $w$ es el período de las franjas de Lau. Asimismo, en Jinhong, 1987 se determinó que la visibilidad de las franjas varía periódicamente en función de la distancia entre las redes fuente-objeto y de la modulación de fase de acuerdo con:

$$
V=4 \sum_{n= \pm 1, \pm 3 \ldots}\left|C_{n}\right|^{2} \operatorname{sen}(m) \cdot \operatorname{sen}\left(\pi \lambda z_{0} n^{2} / d^{2}\right)
$$

Cuando se emplea una red objeto de fase, el contraste óptimo se observa en los planos a las distancias $z_{0}=\frac{\left(M \pm \frac{1}{2}\right) d^{2}}{\lambda}$ $(\mathrm{M}=1,2,3 \ldots)$. Por otra parte, el valor de visibilidad será máximo cuando la modulación toma el valor $m=\pi / 2$. A su vez, en $z_{0}=\frac{M d^{2}}{\lambda}$, la visibilidad será mínima mientras que para $z_{0}=\frac{\left(M \pm \frac{1}{4}\right) d^{2}}{\lambda}$ las franjas presentarán una visibilidad reducida con respecto a la que se presenta para $z_{0}=\frac{\left(M \pm \frac{1}{2}\right) d^{2}}{\lambda}$.

A continuación, se analizará lo que ocurre cuando se emplea una red almacenada en un cristal fotorrefractivo como red objeto. Debemos notar que las variaciones de intensidad incidente en un cristal fotorrefractivo se traducen en cambios en el índice de refracción. Por lo tanto, la información de entrada queda codificada como información de fase. En el caso de un cristal fotorrefractivo BSO la modulación de fase alcanza valores típicos entre $10^{-4}$ y $10^{-5}$.

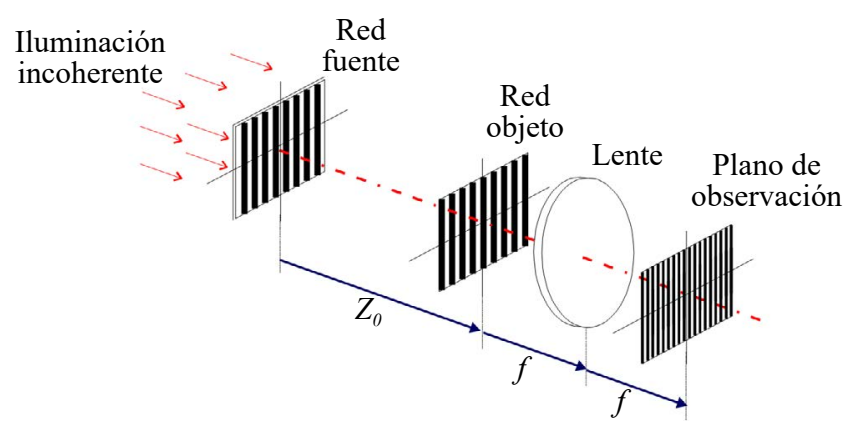

Figura 1. Dispositivo experimental de Lau 
Como se mencionó anteriormente, cuando se emplea una red objeto de fase, la visibilidad de las franjas de Lau depende de la modulación. En la figura 2 se muestran los perfiles de intensidad de las franjas obtenidos teóricamente, considerando redes objeto planas de fase y con modulaciones de $10^{-4} \mathrm{y}$ de $\pi / 2$. Para $m=\pi / 2$ se observan franjas de máxima visibilidad; en cambio, cuando se consideran modulaciones del orden de las obtenidas con cristales BSO, se observa que la visibilidad de las franjas decae considerablemente.

La figura 3 muestra las franjas de Lau obtenidas experimentalmente a partir de una red de fase almacenada en un cristal BSO. Es evidente la alta visibilidad de las franjas obtenidas en comparación con el resultado simulado de la figura 2 (a). Nótese sin embargo que la simulación fue realizada considerando una red plana. Este es el punto de partida para profundizar el efecto de volumen de la red objeto en el fenómeno de Lau.

\section{Descripcion del esquema experimental}

En Ref. (Forte, 2012) se analizó la influencia del volumen del medio fotorrefractivo en la visibilidad de las autoimágenes. Se propuso reemplazar el parámetro de modulación $m$ por un nuevo parámetro de fase $\beta_{\text {mod }}$, que tenga en cuenta las variaciones de modulación en el volumen del medio de registro. En este trabajo, dichas ideas se extienden al dispositivo de Lau y, en las ecuaciones (2) y (3), se reemplaza la modulación $m$ por $\beta_{\text {mod }}$. Finalmente, se estudian las franjas para distintas distancias $z_{0}$ entre las redes fuente-objeto.

La figura 4 muestra el esquema experimental correspondiente a la etapa de registro de la red fotorrefractiva. La imagen de una red de Ronchi de baja frecuencia, G, iluminada incoherentemente es proyectada dentro del volumen de un cristal $\mathrm{B}_{12} \mathrm{SiO}_{20}$ (conocido como BSO). Un filtro interferencial, $F_{G}$, centrado en la longitud de onda $\lambda_{\mathrm{G}}=534$ $\mathrm{nm}\left(\Delta \lambda_{\mathrm{G}}= \pm 7 \mathrm{~nm}\right)$, es usado en la etapa de registro debido a la alta sensibilidad del cristal en la región azul-verde del espectro visible. La lente $\mathrm{L}_{2}$, de distancia focal $\mathrm{f}=50 \mathrm{~mm}$, forma una imagen de la red en el cristal con magnificación unitaria. Para nuestra propuesta, se utiliza un cristal BSO en configuración transversal. Las dimensiones de los cristales considerados son $\mathrm{L}_{\mathrm{X}}=10 \mathrm{~mm}$ y $\mathrm{L}_{\mathrm{Y}}=10 \mathrm{~mm}$, mientras que $\mathrm{L}_{z}$ es variable. Un campo eléctrico se aplica al cristal en una dirección paralela al vector de onda de la imagen de la red proyectada y un diafragma $\mathrm{P}$ se ubica detrás de la lente formadora de imagen $\mathrm{L}_{2}$. En nuestras condiciones experimentales, el diámetro de la pupila de salida del sistema óptico, controlado por el diafragma $P$, se mantiene fijo en un valor que asegure el registro de red en el volumen completo del cristal. La distribución de intensidad luminosa 3D incidente en el medio de registro es mapeada, vía el efecto fotorrefractivo, como una perturbación de índice de refracción (Tebaldi, 1998, Huignard, 1989), generando de este modo una red de fase de volumen con el mismo vector de onda que la red plana de entrada.

En la etapa de generación de las franjas de Lau, la red fuente de amplitud $G_{A}$ es iluminada con una fuente

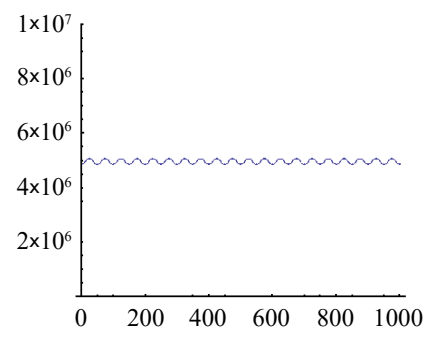

(a)

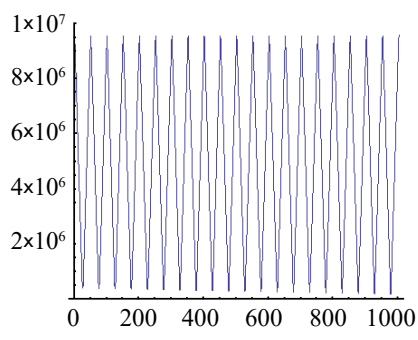

(b)
Figura 2. Perfil de intensidad de los patrones de Lau simulados, correspondientes a una red objeto plana de fase con una modulación (a) $m=10^{-4}$ y (b) $m=\pi / 2$

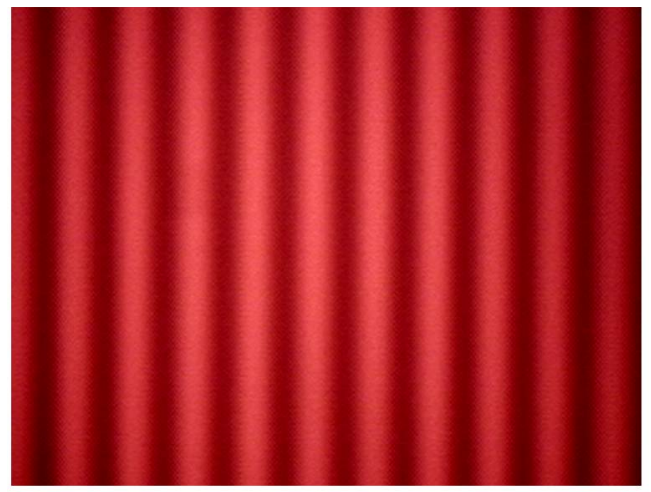

Figura 3. Franjas de Lau obtenidas empleando como red objeto una red almacenada en un cristal fotorrefractivo tipo BSO

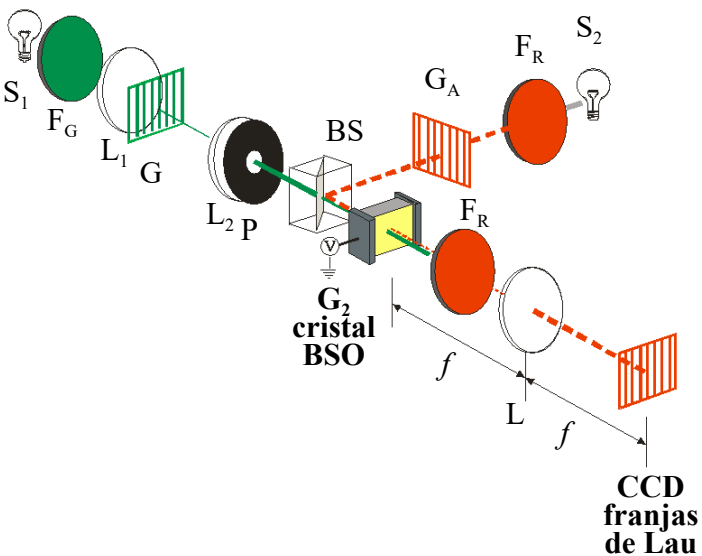

Figura 4. Esquema experimental $\mathrm{S}_{1}$ y $\mathrm{S}_{2}$ : fuentes incoherentes, $\mathrm{F}_{\mathrm{G}}$ y $\mathrm{F}_{\mathrm{R}}$ : filtros interferenciales centrados en $\lambda_{G=} 534 \mathrm{~nm}\left(\Delta \lambda_{G}= \pm 7 \mathrm{~nm}\right)$, y $\lambda_{R}=633 \mathrm{~nm}\left(\Delta \lambda_{R}= \pm 5 \mathrm{~nm}\right)$, respectivamente, $\mathrm{L}_{1}, \mathrm{~L}_{2}, \mathrm{~L}$ : lentes, BS:

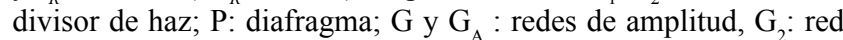
objeto almacenada en el cristal fotorrefractivo como modulación de fase, CCD (cámara CCD)

incoherente provista con un filtro interferencial $F_{R}$ con su máximo de transmisión en $\lambda_{R}=633 \mathrm{~nm}(\Delta \lambda \mathrm{R}= \pm 5 \mathrm{~nm})$. Esta longitud de onda de lectura está fuera del rango de sensibilidad espectral del cristal BSO, evitando así la degradación de 
la información almacenada en la etapa de registro descrita anteriormente. $\mathrm{La}$ red $\mathrm{G}_{\mathrm{A}}$ está separada una distancia $\mathrm{z}_{0}$ de la red $\mathrm{G}_{2}$, que actúa como red objeto del dispositivo de Lau. Esta última se registra en el medio fotorrefractivo de volumen cuando se forma la imagen de la red de amplitud $\mathrm{G}$ en el cristal. La lente $L$ realiza la transformada de Fourier de la distribución luminosa proveniente de $\mathrm{G}_{\mathrm{A}}$ y transmitida a través de la red fotorrefractiva $\mathrm{G}_{2}$, formándose así las franjas de Lau en el plano de Fourier de la lente L. A la salida del cristal se coloca un segundo filtro interferencial $F_{R}$ con su máximo de transmisión en $\lambda_{R}=633 \mathrm{~nm}(\Delta \lambda \mathrm{R}= \pm 5 \mathrm{~nm})$, para bloquear la luz proveniente de la etapa de registro y la imagen que esta pueda formar de la red.

\section{Resultados y discusión}

Como se reportó en Forte 2012, la generación de la red de fase de volumen depende de la profundidad de foco de la imagen registrada, de la amplitud del campo de cargas espaciales y del espesor del medio de volumen de registro. La profundidad de foco de la imagen proyectada se controla cambiando el diámetro de la pupila de salida del sistema óptico de registro. De esta manera, se modifica la región en la cual la red de índice se extiende dentro del cristal. En el análisis que realizaremos a continuación, el diámetro de la pupila permanecerá fijo en un valor que asegure el registro de la red en el volumen completo del cristal.

Por otra parte, la amplitud de modulación de la red de índice depende de la amplitud del campo de carga espacial, el cual es controlado mediante el campo eléctrico externo aplicado al cristal tipo silenita. Este parámetro también se mantendrá sin variaciones en nuestro análisis.

En las figuras 5 y 6 , se presentan los perfiles de intensidad de los patrones de Lau correspondientes a dos distancias $z_{0}$, una asociada a la máxima visibilidad y otra a una visibilidad reducida. Estos perfiles fueron calculados usando las redes de volumen simuladas (Forte, 2012, Forte, 2011), en las condiciones del arreglo experimental de la figura 4. En estas condiciones, la modulación $m$ correspondiente a una red plana es reemplazada por el parámetro de modulación, $\beta_{\text {mod }}$, dado por:

$$
\beta_{\text {mod }}=k_{r}\left(\int_{0}^{L_{z}}\left(\Delta n\left(z^{\prime}, 0 ; E_{0}, D, L_{z}\right)-\Delta n\left(z^{\prime}, d / 2 ; E_{0}, D, L_{z}\right)\right) d z^{\prime}\right)
$$

donde $\mathrm{k}_{\mathrm{r}}$ es el número de onda, $\Delta n$ es la variación del índice de refracción, $\mathrm{E}_{0}$ es el campo externo aplicado, $D$ es el diámetro de la pupila $\mathrm{P}, \mathrm{L}_{\mathrm{z}}$ es el espesor del cristal. A continuación, solo se analizará el efecto del parámetro $\mathrm{L}_{Z}$.

Los perfiles de las franjas de Lau de la figura 5 corresponden a una distancia entre las redes fuente-objeto de $z_{0}=\left(\frac{3}{2}\right) \frac{d^{2}}{\lambda_{R}}$. Asimismo, la red objeto es generada en cristales BSO con diferentes valores del espesor $\mathrm{L}_{z}$. A partir de los resultados es evidente que el contraste de las franjas varía en términos de los espesores considerados. En particular, se observa un aumento paulatino del contraste de las franjas entre los casos de $1 \mathrm{~mm}$ y $6 \mathrm{~mm}$.

La ecuación (3) muestra que la visibilidad de las franjas de Lau varía sinusoidalmente en función del parámetro de

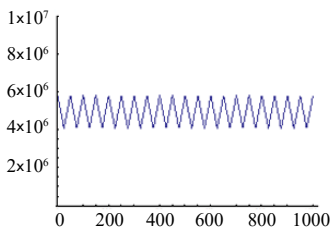

(a)

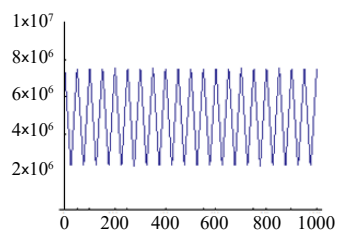

(c)

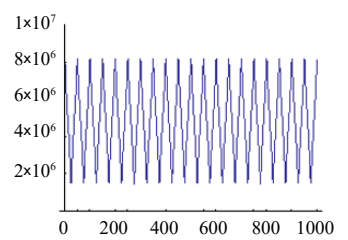

(e)

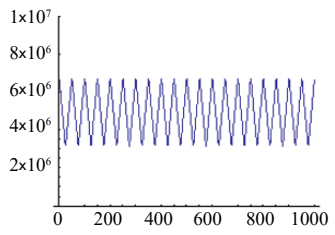

(b)

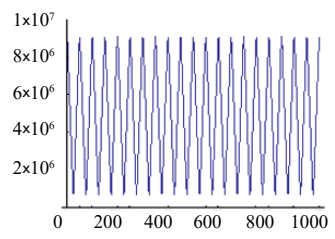

(d)

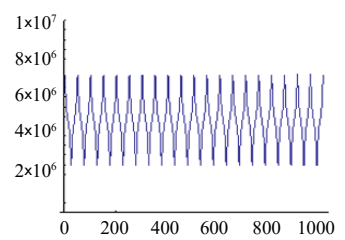

(f)
Figura 5. Perfiles de intensidad de las franjas de Lau correspondientes a una distancia entre redes de $z_{0}=\left(\frac{3}{2}\right) \frac{d^{2}}{\lambda_{R}}$ y generados a partir de una red fotorrefractiva de volumen con espesores (a) $\mathrm{L}_{\mathrm{z}}=1 \mathrm{~mm}$ (b) $\mathrm{Lz}=2 \mathrm{~mm}$ (c) $\mathrm{Lz}=3 \mathrm{~mm}$ (d) $\mathrm{Lz}=6 \mathrm{~mm}$ (e) $\mathrm{Lz}=12 \mathrm{~mm}$ y (f) $\mathrm{Lz}=14 \mathrm{~mm}$

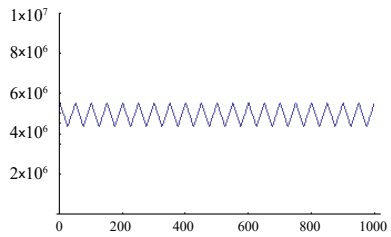

(a)

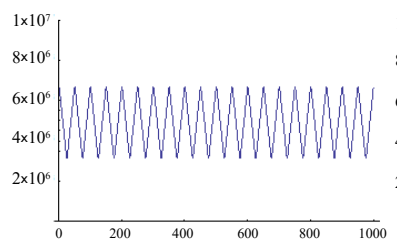

(c)

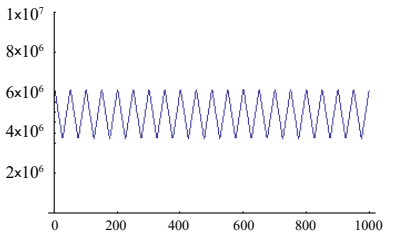

(b)

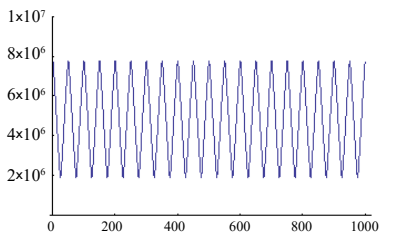

(d)
Figura 6. Perfiles de intensidad de las patrones correspondientes a una distancia entre redes de $z_{0}=\left(\frac{3}{4}\right) \frac{d^{2}}{\lambda_{R}}$ y generados a partir de una red fotorrefractiva de volumen con espesores (a) $\mathrm{L}_{\mathrm{z}}=1 \mathrm{~mm}$ (b) $\mathrm{Lz}$ $=2 \mathrm{~mm}$ (c) $\mathrm{Lz}=3 \mathrm{~mm}$ (d) $\mathrm{Lz}=6 \mathrm{~mm}$

modulación. En nuestra propuesta, el parámetro de modulación está dado por $\beta_{\text {mod }}$ que depende del espesor de la red fotorrefractiva. La visibilidad máxima para una red de fase se obtiene con una modulación $\beta_{\bmod }=\pi / 2$. En el caso planteado ese máximo corresponde a un espesor de la red de volumen o espesor de cristal de aproximadamente 7,5 $\mathrm{mm}$. En consecuencia, para espesores mayores la visibilidad decae, lo que se visualiza en los perfiles de la figura 5 (e) y 5 (f). 
Como se mencionó anteriormente, en los planos $z_{0}=\frac{\left(M \pm \frac{1}{4}\right) d^{2}}{\lambda_{R}}$ las franjas presentarán una visibilidad reducida con respecto a la que se presenta en $z_{0}=\frac{\left(M \pm \frac{1}{2}\right) d^{2}}{\lambda_{R}}$. Esto puede ser confirmado considerando los resultados de la figura 6 donde se observa una menor visibilidad de franjas en comparación con los mismos espesores de cristal que en la figura 5.

\section{Conclusiones}

En este trabajo se estudió el efecto Lau mediante el uso de una red de fase de volumen generada en un cristal fotorrefractivo. La distribución de intensidad 3D almacenada en el cristal se genera al formar la imagen de una red de Ronchi incoherentemente iluminada en un cristal BSO. Se empleó una pupila adecuada con el propósito de garantizar la generación de una red en el volumen completo del cristal. Esta distribución es mapeada como una perturbación de índice de refracción a través del efecto fotorrefractivo, generando una red de fase de volumen. En el experimento de Lau convencional el control de la visibilidad de las franjas depende de la distancia entre las redes fuente-objeto, $\mathrm{z}_{0}$. En nuestra propuesta, se incorpora el espesor del cristal como un parámetro adicional de control de la visibilidad para cualquier distancia $\mathrm{z}_{0}$. Esto se verificó con el análisis de las franjas para $z_{0}=\left(\frac{3}{2}\right) \frac{d^{2}}{\lambda_{R}}$ y $z_{0}=\left(\frac{3}{4}\right) \frac{d^{2}}{\lambda_{R}}$. Asimismo, la visibilidad de las franjas mantiene la dependencia sinusoidal de la red de fase plana, pero introduciendo un parámetro de fase apropiado que depende del espesor de la red fotorrefractiva de volumen.

\section{Agradecimientos}

Este trabajo fue auspiciado por CONICET No. 0849/16 (Argentina); Facultad de Ingeniería, Universidad Nacional de La Plata No. 11/I215 y 11/I239 (Argentina).

\section{Contribución de los autores}

Los autores mencionados anteriormente han contribuido de manera original y eficiente para el desarrollo completo del artículo.

El trabajo fue presentado en la modalidad de póster en el XV Encuentro Nacional de Óptica y VI Conferencia Andina y del Caribe en Óptica y sus aplicaciones (ENO-CANCOA), evento llevado a cabo en la Universidad Industrial de Santander sede Bucarica, en Bucaramanga, Colombia del 20 al 24 de noviembre de 2017.

\section{Conflicto de intereses}

No existen conflictos de intereses que puedan influir en la transparencia $u$ objetividad en el proceso de revisión por pares y publicación.

\section{Referencias}

Patorski, K. (1989). The self-imaging phenomenon and its applications.Prog. Opt., 27: 3-108.

Lau, E. (1948). Beugungserscheinungen and Doppelrastern. Ann. Phys. (Leipzig). 6: 417-423.

Jahns J., Lohmann, A. W. (1979). The Lau effect a diffraction experiment with incoherent illumination. Opt. Commun. 28: 263-267.

Gori, F. (1979). Lau effect and coherence theory. Opt. Commun., 31: 4-8.

Brenner, K. H., Lohmann, A. W., Ojeda- Castañeda, J. (1983). Lau effect: OTF theory. Opt. Commun., 46: 14-17.

Colautti, C., Sicre, E.E., Garavaglia, M. (1988). Analysis of the Lau effect using Walsh functions. Opt. Lett., 13: 1069-1071.

Swanson, G. J., Leith, E. N. (1982). Lau effect and grating imaging. J. Opt. Soc. Am., 72: 552-555.

Swanson, G. J., Leith E. N. (1985). Analysis of the Lau effect and generalized grating imaging. J. Opt. Soc. Am., A, 2: 789-793.

Patorski, K. (1983). Incoherent superposition of multiple selfimaging. Lau effect and moiré fringe explanation.Optica Acta, 30: 745-748.

Tebaldi, M., Angel Toro, L., Bolognini, N. (1999). Interferometry based on Lau effect with a grating registered in a photorefractive crystal.Optics \& Laser Technology, 31: 127-134.

Angel, L., Tebaldi, M., Henao,R. (1999). Phase stepping in Lau interferometry. Opt. Commun., 164: 247-255.

Jinhong, T. (1987). Theoretical analysis of the Lau effect with a sinusoidal phase grating.J. Mod. Opt. 34: 307-313.

Jinhong, T. (1988). The diffraction near fields and Lau effect of a square-wave modulated phase grating.J. Mod. Opt., 35: 1399-1408.

Forte, G., Lencina, A., Tebaldi, M., Bolognini, N. (2012). Talbot effect by a photorefractive volume phase grating.Appl. Opt., 51: 479-485.

Forte, G., Lencina, A., Tebaldi, M., Bolognini, N. (2011). Selfimaging by a volume grating.Opt. Commun., 284: 24942499.

Tebaldi, M., Angel, L., Bolognini,N. (1998) Lau fringes formation using a grating registered in a BSO crystal. Opt. Eng., 37: 2971-2978.

Huignard, P., Gunter, P. (1989). Optical processing using wave mixing in photorefractive crystals. En P. Gunter, J. P. Huignard (Eds.), Photorefractive Material and their Applications II (205-273) Berlin and Heidelberg, Alemania: Springer- Verlag.

Forte G, Tebaldi M, Bolognini N. (2017) Study of Lau fringes generated by a photorefractive volume grating, Opt. Commun., 396: 110-115. 